\title{
Preface to Special Issue on Dynamic Games for Modeling and Control of Epidemics
}

\author{
Quanyan Zhu' ${ }^{1}$ Elena Gubar ${ }^{2} \cdot$ Eitan Altman $^{3}$
}

Accepted: 27 January 2022 / Published online: 10 February 2022

(c) The Author(s), under exclusive licence to Springer Science+Business Media, LLC, part of Springer Nature 2022

\begin{abstract}
This preface introduces the special issue on Dynamic Games for Modeling and Control of Epidemics. It showcases 12 papers with timely contributions to dynamic games and their applications to the modeling, analysis, and control of epidemics. The papers in this collection connect dynamic games and epidemic models to address the recent challenges related to screening, containment, and mitigation strategies for epidemics. This collection covers broad application areas in networks, human behaviors, and epidemiology as well as a diverse range of dynamic game methods, including evolutionary games, differential games, and mean-field games.
\end{abstract}

The recent COVID-19 pandemic has caused a significant social and economic disruption in today's connected world. There is an imminent need to understand and control the spreading of the disease over networks. Dynamic games provide a natural framework to model and analyze the individual incentives and their social interactions over large networks. Sophisticated models such as evolutionary games [9, 15] and mean-field games [3, 13] have enabled the understanding of the emerging population-level phenomena and effective control mechanisms. Connecting dynamic games and epidemic models offers a scientific foundation for rigorous and quantitative analysis and design of screening, containment, and mitigation strategies for large-scale dynamic and network systems. This cross-disciplinary approach will not only address the current challenges with COVID-19 but also shed light on related

This article is part of the topical collection "Modeling and Control of Epidemics" edited by Quanyan Zhu, Elena Gubar and Eitan Altman.

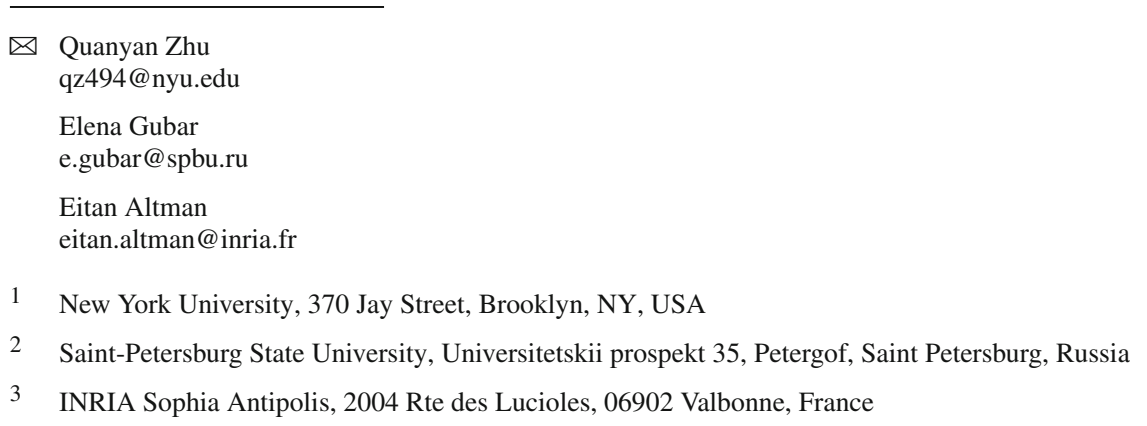


problems in the domain of cybersecurity [7, 8, 10], wireless communications [2, 12, 14], Internet of Things [4-6], and social networks [1, 11, 16].

This special issue showcases timely contributions to dynamic games and their applications to the modeling, analysis, and control of epidemics. It includes twelve papers, featuring one review paper that comprehensively introduces the application of game theory in epidemic control and ten original research papers that deal with many facets of the epidemics and decision-making. The research papers in this collection contribute to three major research areas. The first area is the emerging theory of differential and stochastic game models and tools for epidemics. The second one is the novel applications of evolutionary game models and theory for the control and analysis of epidemics. The third area is the novel applications of dynamic games for social distancing policies to control the spreading of epidemics. Many papers in this special issue are motivated by the recent ongoing COVID-19 pandemic. It has become increasingly important for both scientific understandings of pandemic control and the development of effective control policies.

The first article is a review paper that provides an overview of game-theoretic frameworks for epidemic modeling and decision-making. In the article entitled "Game-Theoretic Frameworks for Epidemic Spreading and Human Decision-making: A Review" by Y. Huang et al., the authors provide a review of different epidemic models and their applications. Their study is motivated by the recent significant growth of the game-theoretical and making-decision models for the epidemic modeling. They present a classification of the existing models which integrate human decisions into the epidemic propagation in the populations. The review indicates that three models are unique among a good number of the relevant works. The authors present their special model formulation, solution techniques, and results. Building on the recent advances, the authors identify several open problems which can be studied further.

Following the review article, the next four papers contribute to the differential games and stochastic games for the applications of epidemic control. In the article entitled "Finite State Graphon Games with Applications to Epidemics" by R. Carmona et al., the authors present a graphon-based mean-field model that captures the heterogeneous interactions of non-identical players over networks. This work creates a novel mathematical framework of epidemic processes over graphon-based networks and develops a new class of dynamic game models in which players make decisions by themselves. The authors show the existence of Nash equilibrium solutions and provide sufficient conditions that characterize them. The equilibrium solutions rely on solving a continuum of fully coupled forward-backward ordinary differential equations. This work leverages tools from machine learning to create numerical methods and algorithms to find equilibrium solutions for different compartmental models in epidemiology.

In the article entitled "A Partially Observable Stochastic Zero-sum Game for a Network Epidemic Control Problem" by Y. Hayel et al., the authors investigate an information security application of the epidemic models. They design a novel zero-sum partially observable stochastic game to describe the interaction between a network defender and an attacker. It is formulated to investigate the optimal protective strategies against virus propagation, which is modeled by compartmental epidemic models. The main result of this work is a formal proof of convergence of the value backup operator. The novel framework leads to several directions for future research, including the study of optimal attack and defense strategies, taking into account the properties of network structures.

In the article entitled "Mobility Choices and Strategic Interactions in a Two-Group Macroeconomic-Epidemiological Model" by D. La Torre, the author aims to study the heterogeneous impacts on different population groups by establishing a two-group differential game in which the interactions between groups determine the overall prevalence of an infec- 
tious disease, which in turn affects the level of economic activity. Individuals may reduce their mobility to contain the disease spreading but have an incentive to free ride. This work characterizes the cooperative and noncooperative equilibria of the game and quantifies the inefficiency induced by the failure of single groups to internalize the externality. The longrun eradication of the disease is shown to be possible even in the absence of coordination, and the coordination leads to a faster reduction in the infected population size in finite time. The authors indicate that the generalization of the proposed framework by including factors such as disease-induced mortality and heterogeneity in the disease transmission can be a promising next step to provide real-world policy recommendations for COVID-19.

In the article entitled "Game-Theoretical Model of the Voluntary Use of Insect Repellents to Prevent Zika Fever" by J. Rychtar, the author investigates the Zika virus and its epidemic process. The virus can spread to people through mosquito bites primarily in tropical and subtropical areas of the world. Motivated by the recent 2015-2016 epidemic of Zika fever, the authors created mathematical models to understand the interactions among the population, individuals, and the environment. Building on the dynamic system models, the authors use a game-theoretic approach to understand the impact of the voluntary use of DEET, a chemical ingredient used for insect repellents, to prevent the spread of the Zika virus when there are no specific treatments or vaccines available. The game is a repeated population game where the payoff of individual players depends on their choice of adoption of DEET and the average strategy used by the population. The results show that the optimal use of the DEET can effectively eliminate the disease and the conclusion does not depend on the cost of the insect repellent. This work

The next three articles contribute to the evolutionary game theory in connection with epidemics. In the article entitled "The Mask Game with Multipopulations" by E. Altman et al., the authors are motivated by the recommendations of mask-wearing from public health organizations and propose a mask game in which players decide whether to wear a mask or not according to their individual risk state and health state. The mask game is formulated as a Bayesian population game. The authors leverage the tools from evolutionary games and obtain results of the equilibrium of the cases of single populations and multiple populations. Results from this work provide a quantitative understanding of the impact of voluntary maskwearing on the epidemic spreading. The authors suggest several directions of future works including the study of strain games in which the new variants of viruses compete with each other for survival and the competitive vaccination games between countries and cities where interdependent regions develop their own health policies to fight against the pandemic.

In the article entitled "COVID-19 and Stigma: Evolution of Self-Restraint Behavior" by K. Kurita, the authors are motivated by the recent policies by countries, such as Japan and Sweden, to fight against COVID-19. The policies reply on individual self-restraint without enforcement. They have been proven to be more successful in controlling the number of infections than in other Organisation for Economic Co-operation and Development (OECD) countries. This work aims to understand the impact of imposed social stigma on the containment of disease spreading. The authors create a framework based on replicator dynamics to analyze the interplay among self-restraint behavior, social stigma, and the virus spreading. The analysis of the steady states of the dynamics leads to the explanation of how nonlegally binding policy reduces social activities. The further comparative analysis shows that increasing the stigma cost does not necessarily further reduce social mobility. This work demonstrates that social stigma plays an important role in containing COVID-19, but the authors also note that extreme stigmatization can have a negative impact which leads to discrimination, prejudice, and violence. 
In the article entitled "Herd Behaviors in Epidemics: A Dynamics-Coupled Evolutionary Games Approach" by S. Liu et al., the authors are inspired by the recent COVID-19 pandemic, which has witnessed a strong interplay between human behaviors and the spreading of the pandemic. The severity of the pandemic will lead to individual social activity levels, which in turn affects the spreading of the pandemic. The authors use the degree-based mean-field epidemic model over a complex network to capture the epidemic spreading processes and use evolutionary game dynamics to capture the adaptive human behaviors. The two dynamics are coupled to understand the confluences between the two and study how the social choices of the individuals impact the epidemic process. This result further formulates an equivalent finite-player game and an equivalent network to represent the interactions among the finite number of subpopulations. Numerical experiments based on New York City data are used to provide a match between models and the data.

The last four papers deal with social distancing in epidemic control and contribute to both the theory and applications of the problem. The authors have developed new frameworks interconnecting human behaviors, decision-making, epidemic modeling, and systems and control. In the article entitled "Dynamic Games of Social Distancing During an Epidemic: Analysis of Asymmetric Solutions" by I. Kordonis et al., the authors propose a dynamic game model with a continuum of players to study the impact of social distancing behaviors on epidemics. The players have uncertainties on their infection state, which evolves according to a variant of Susceptible-Infected-Recovered (SIR) dynamics and choose actions based on limited information. This work first shows the existence of Nash equilibria of the proposed game framework and uses a class of nonlinear complementarity problems to characterize them. The authors exploit the monotonicity properties of the optimal policies and reduce the computation complexity of the Nash equilibrium using a low-dimensional optimization problem. It is observed from numerical experiments that homogeneous agents can have different behaviors and stringent constraints on the actions can benefit the more vulnerable agents at the expense of the less vulnerable ones.

In the article entitled "Necessity of Social Distancing in Pandemic Control: A Dynamic Game Theory Approach" by I. Dahmouni and K. Kanani, the authors create a dynamic game to model society with healthy and vulnerable individuals, who decide whether to participate in in-person activities based on the severity of the epidemic. A local government controls the epidemics by imposing social distancing regulations that adapt to the infection status of the society. This work has considered four scenarios of society in which individuals can be non-cooperative, cooperative, egoistic, or altruistic. The authors show that the steady-state of the epidemic under the altruistic scenario where individuals put the needs of the vulnerable first stands out as the best among the four. This work creates variants of the model and the impact of individual responses to the epidemics in a society. It offers insights into the role of human behaviors in the way that we fight against the pandemic we are experiencing at the moment.

In the article entitled "Epidemic Spreading and Equilibrium Social Distancing in Heterogeneous Networks" by H. Amini, the author studies the impact of social distancing using a SIR epidemic process with multiple types of subpopulations that interact over a network. This work establishes the epidemic reproduction number for different social distancing strategies as an important metric to identify network vulnerability and inform vaccination policies. An effective policy should result in the reproduction number smaller than one. Building on this result, the author further studies a social distancing game in which individuals choose their social distancing levels based on the global infection status. This work shows that there exists a performance gap between the voluntary social distancing solution and the social optimum 
solution. The author points out several research directions including the investigation of the individual choice of connectivity and social planning under a limited supply of vaccines.

In the article entitled "Social Distancing, Gathering, Search Games: Mobile Agents on Simple Networks" by S. Alpern et al., the authors are motivated by the social distancing policies in the recent COVID-19 pandemic. Individuals are required to be kept two meters apart. This work studies the social distancing game on a connected graph where agents adopt a lazy random walk and choose the probability to move to a random adjacent node to achieve the goal of keeping some distance apart. The authors consider various graph structures and different knowledge patterns. The results characterize the equilibrium solution of the game and show that there are no symmetric equilibria in some cases. The authors note that this work also extends the existing literature on predator-prey search games to multiple searchers by allowing distinct laziness for two types of agents.

Even though this collection is not exhaustive in the choice of topics within the field of dynamic games and their applications, it does cover a variety of areas and recent developments on the topic of current interest. It should be useful to researchers in differential and dynamic games, systems and control, network science and engineering, epidemiology, and public health policy. We are hopeful that this collection will spawn an increasing level of interest and cross-disciplinary collaborations across multiple research areas. We thank the authors for their contributions to this collection and the reviewers who have provided valuable comments to improve the quality of this special issue.

\section{References}

1. Altman E, Jain A, Shimkin N, Touati C (2016) Dynamic games for analyzing competition in the internet and in on-line social networks. In: International conference on network games, control, and optimization, pp 11-22. Springer

2. Altman E, Neglia G, De Pellegrini F, Miorandi D (2009) Decentralized stochastic control of delay tolerant networks. In: IEEE INFOCOM 2009, pp 1134-1142. IEEE, Springer

3. Carmona R, Delarue F (2018) Probabilistic theory of mean field games with applications. I, volume 83 of probability theory and stochastic modelling

4. Farooq J, Zhu Q (2021) Resource management for on-demand mission-critical internet of things applications. Wiley

5. Farooq MJ, Zhu Q (2018) On the secure and reconfigurable multi-layer network design for critical information dissemination in the internet of battlefield things (IoBT). IEEE Trans Wirel Commun 17(4):2618-2632

6. Farooq MJ, Zhu Q (2019) Modeling, analysis, and mitigation of dynamic botnet formation in wireless IoT networks. IEEE Trans Inf Forens Secur 14(9):2412-2426

7. Gubar E, Taynitskiy V, Zhu Q (2018) Optimal control of heterogeneous mutating viruses. Games 9(4):103

8. Hayel Y, Zhu Q (2017) Epidemic protection over heterogeneous networks using evolutionary Poisson games. IEEE Trans Inf Forens Secur 12(8):1786-1800

9. Hofbauer J, Sigmund K et al (1998) Evolutionary games and population dynamics. Cambridge University Press

10. Huang Y, Zhu Q (2019) A differential game approach to decentralized virus-resistant weight adaptation policy over complex networks. IEEE Trans Control Netw Syst 7(2):944-955

11. Jiang C, Chen Y, Liu KR (2014) Graphical evolutionary game for information diffusion over social networks. IEEE J Sel Top Signal Process 8(4):524-536

12. Khouzani M, Sarkar S, Altman E (2012) Maximum damage malware attack in mobile wireless networks. IEEE/ACM Trans Netw 20(5):1347-1360

13. Lasry JM, Lions PL (2007) Mean field games. Jpn J Math 2(1):229-260

14. Tembine H, Altman E, El-Azouzi R, Hayel Y (2009) Evolutionary games in wireless networks. IEEE Trans Syst Man Cybern Part B Cybern 40(3):634-646

15. Weibull JW (1997) Evolutionary game theory. MIT Press 
16. Zhu Q, Clark A, Poovendran R, Başar T (2013) Deployment and exploitation of deceptive honeybots in social networks. In: 52nd IEEE conference on decision and control, pp 212-219. IEEE

Publisher's Note Springer Nature remains neutral with regard to jurisdictional claims in published maps and institutional affiliations. 